\title{
Chronic treatment with C-type natriuretic peptide impacts differently in the aorta of normotensive and hypertensive rats
}

\author{
Pflügers Archiv - European Journal of Physiology
}

pp 1-13 | Cite as

- Carolina Caniffi (1) (2) Email author (ccaniffi@ffyb.uba.ar)View author's OrcID profile (View OrcID profile)

- Flavia M. Cerniello (1) (2)

- Gonzalo Bouchet (1) (2)

- María L. Sueiro (1) (2)

- Analía Tomat (1) (2)

- Daniel González Maglio (3) (4)

- Jorge E. Toblli (5)

- Cristina Arranz (1) (2)

1. Universidad de Buenos Aires, Facultad de Farmacia y Bioquímica, Cátedra de Fisiología, , Buenos Aires, Argentina

2. CONICET - Universidad de Buenos Aires, Instituto de la Química y Metabolismo del Fármaco (IQUIMEFA), , Buenos Aires, Argentina

3. Universidad de Buenos Aires, Facultad de Farmacia y Bioquímica, Cátedra de Inmunología, , Buenos Aires, Argentina

4. CONICET - Universidad de Buenos Aires, Instituto de Estudios de la Inmunidad Humoral (IDEHU), , Buenos Aires, Argentina

5. Laboratorio de Medicina Experimental, Hospital Alemán, , Buenos Aires, Argentina

Organ physiology

First Online: 11 June 2019

- 77Downloads

Part of the following topical collections:

1. Organ Physiology

\section{Abstract}

The aim of this study was to determine whether exogenous administration of C-type natriuretic peptide (CNP) induces functional and morphological vascular changes in spontaneously hypertensive rats (SHR) compared with normotensive rats. Male 12week-old normotensive Wistar and SHR were administered with saline $(\mathrm{NaCl} 0.9 \%)$ or $\mathrm{CNP}(0.75 \mu \mathrm{g} / \mathrm{h} / 100 \mathrm{~g})$ for 14 days (subcutaneous micro-osmotic pumps). Systolic 
blood pressure (SBP) was measured in awake animals and renal parameters were evaluated. After decapitation, the aorta was removed, and vascular morphology, profibrotic markers, and vascular reactivity were measured. In addition, nitric oxide (NO) system and oxidative stress were evaluated. After 14-days of treatment, CNP effectively reduced SBP in SHR without changes in renal function. CNP attenuated vascular remodeling in hypertensive rats, diminishing both profibrotic and proinflammatory cytokines. Also, CNP activated the vascular NO system and exerted an antioxidant effect in aortic tissue of both groups, diminishing superoxide production and thiobarbituric acid-reactive substances, and increasing glutathione content. These results show that chronic treatment with $\mathrm{CNP}$ attenuates the vascular damage development in a model of essential hypertension, inducing changes in fibrotic, inflammatory, oxidative, and NO pathways that could contribute to beneficial longterm effects on vascular morphology, extracellular matrix composition, and function. The knowledge of these effects of CNP could lead to improved therapeutic strategies to not only control BP but also reduce vascular damage, primarily responsible for the risk of cardiovascular events.

\section{Keywords}

Blood pressure Nitric oxide Oxidative stress Natriuretic peptides

Vascular remodeling Fibrosis

Dr. Jorge E. Toblli passed away in 2018

This is a preview of subscription content, $\underline{\log \text { in }}$ to check access.

\section{Notes}

\section{Acknowledgments}

The authors thank Sandra Landín for secretarial work, Gabriela Noceti for technical assistance, and Ana Borthwick for proofreading and language assistance.

\section{Author contribution}

- Conceived of or designed study: Carolina Caniffi, Cristina Arranz

-Performed research: Carolina Caniffi, Flavia M. Cerniello, Gonzalo Bouchet, María L. Sueiro, Analía Tomat, Daniel González Maglio, Jorge E. Toblli

-Analyzed data: Carolina Caniffi, Flavia M. Cerniello, Daniel González Maglio, Jorge E. Toblli

-Contributed new methods or models: Carolina Caniffi, Analía Tomat, Jorge E. Toblli, Cristina Arranz

-Wrote the paper: Carolina Caniffi, Analía Tomat, Cristina Arranz 


\section{Funding}

This study was funded by Universidad de Buenos Aires (UBA) [UBACYT: 200-20100100060]; Consejo Nacional de Investigaciones Científicas y Técnicas (CONICET) [PIP112-201101-00581]; and Instituto de Química y Metabolismo del Fármaco (IQUIMEFA), UBA-CONICET, Argentina.

\section{Compliance with ethical standards}

\section{Conflict of interest}

The authors declare that they have no conflict of interest.

\section{Ethical approval}

All procedures performed in studies involving animals were in accordance with the ethical standards of the institution or practice at which the studies were conducted.

\section{Publisher's note}

Springer Nature remains neutral with regard to jurisdictional claims in published maps and institutional affiliations.

\section{References}

1. Arejian M, Li Y, Anand-Srivastava MB (2009) Nitric oxide attenuates the expression of natriuretic peptide receptor $\mathrm{C}$ and associated adenylyl cyclase signaling in aortic vascular smooth muscle cells: role of MAPK. Am J Physiol Heart Circ Physiol 296(6):H1859-H1867.

https://doi.org/10.1152/ajpheart.01108.2008

(https://doi.org/10.1152/ajpheart.01108.2008)

CrossRef (https://doi.org/10.1152/ajpheart.01108.2008)

Google Scholar (http://scholar.google.com/scholar_lookup?

title=Nitric\%20oxide\%20attenuates\%20the\%20expression\%20of\%2onatriureti Srivastava\&journal=Am\%20J\%20Physiol\%20Heart\%20Circ\%20Physiol\&volum H1867\&publication_year=2009\&doi=10.1152\%2Fajpheart.01108.2008)

2. Barcellos-Hoff MH, Dix TA (1996) Redox-mediated activation of latent transforming growth factor-beta1. Mol Endocrinol 10:1077-1083.

https://doi.org/10.1210/mend.10.9.8885242

(https://doi.org/10.1210/mend.10.9.8885242)

Google Scholar (http://scholar.google.com/scholar_lookup?title=Redoxmediated\%20activation\%20of\%2olatent\%20transforming\%20growth\%2ofactor beta1\&author=MH.\%2oBarcellos- 
Hoff\&author=TA.\%20Dix\&journal=Mol\%2oEndocrinol\&volume=10\&pages=10771083\&publication_year=1996\&doi=10.1210\%2Fmend.10.9.8885242)

3. Caniffi C, Cerniello FM, Gobetto MN, Sueiro ML, Costa MA, Arranz C (2016) Vascular tone regulation induced by C-type natriuretic peptide: differences in endothelium-dependent and -independent mechanisms involved in normotensive and spontaneously hypertensive rats. PLoS One 11(12):e0167817. https://doi.org/10.1371/journal.pone.0167817

(https://doi.org/10.1371/journal.pone.0167817)

CrossRef (https://doi.org/10.1371/journal.pone.0167817)

Google Scholar (http://scholar.google.com/scholar_lookup?

title=Vascular\%2otone\%2oregulation\%2oinduced\%20by\%20C-

type\%2onatriuretic\%2opeptide\%3A\%20differences\%2oin\%20endothelium-

dependent\%20and\%20-

independent\%20mechanisms\%20involved\%20in\%20normotensive\%20and\%2C

4. Caniffi C, Elesgaray R, Gironacci M, Arranz CT, Costa MA (2010) C-type

natriuretic peptide effects on cardiovascular nitric oxide system in

spontaneously hypertensive rats. Peptides 31(7):1309-1318.

https://doi.org/10.1016/j.peptides.2010.03.030

(https://doi.org/10.1016/j.peptides.2010.03.030)

CrossRef (https://doi.org/10.1016/j.peptides.2010.03.030)

Google Scholar (http://scholar.google.com/scholar_lookup?

title=C-type\%2onatriuretic\%2opeptide\%20effects\%20on\%2ocardiovascular\%2

1318\&publication_year $=2010 \& d o i=10.1016 \% 2 F j$.peptides.2010.03.030)

5. Ceron CS, Rizzi E, Guimaraes DA, Martins-Oliveira A, Cau SB, Ramos J, Gerlach RF, Tanus-Santos JE (2012) Time course involvement of matrix metalloproteinases in the vascular alterations of renovascular hypertension.

Matrix Biol 31(4):261-270. https://doi.org/10.1016/j.matbio.2012.01.009

(https://doi.org/10.1016/j.matbio.2012.01.009)

CrossRef (https://doi.org/10.1016/j.matbio.2012.01.009)

Google Scholar (http://scholar.google.com/scholar_lookup?

title=Time\%20course\%20involvement\%20of\%20matrix\%20metalloproteinases

Oliveira\&author $=$ SB.\%20Cau\&author $=$ J.\%20Ramos\&author=RF.\%20Gerlach\&

Santos\&journal $=$ Matrix\%20Biol $\&$ volume $=31$ \&issue $=4$ \&pages $=261-$

270\&publication_year $=2012 \& d o i=10.1016 \% 2 F j$.matbio.2012.01.009)

6. Costa MA, Elesgaray R, Caniffi C, Fellet A, Arranz C (2007) Role of

cardiovascular nitric oxide system in C-type natriuretic peptide effects.

Biochem Biophys Res Commun 359(1):180-186.

https://doi.org/10.1016/j.bbrc.2007.05.095

(https://doi.org/10.1016/j.bbrc.2007.05.095)

CrossRef (https://doi.org/10.1016/j.bbrc.2007.05.095)

Google Scholar (http://scholar.google.com/scholar_lookup?

title=Role\%20of\%20cardiovascular\%2onitric\%200xide\%2osystem\%2oin\%20Ctype\%2onatriuretic\%2opeptide\%2oeffects\&author=MA.\%2oCosta\&author=R.\% 186\&publication_year $=2007 \& d o i=10.1016 \% 2 F j . b b r c .2007 .05 .095)$

7. Crabos M, Coste P, Paccalin M, Tariosse L, Daret D, Besse P, Bonoron-Adèle S (1997) Reduce basal NO-mediated dilation and decreased NO-synthase expression in coronary vessels of spontaneously hypertensive rats. J Mol Cell Cardiol 29:55-65. https://doi.org/10.1006/jmcc.1996.0251

(https://doi.org/10.1006/jmcc.1996.0251)

CrossRef (https://doi.org/10.1006/jmcc.1996.0251) 
Google Scholar (http://scholar.google.com/scholar_lookup?

title=Reduce\%2obasal\%20NO-mediated\%2odilation\%20and\%2odecreased\%20NOsynthase\%20expression\%20in\%20coronary\%20vessels\%20of\%2ospontaneously\%2oh Ad\%C3\%A8le\&journal=J\%20Mol\%20Cell\%20Cardiol\&volume=29\&pages=5565\&publication_year $=1997 \& d o i=10.1006 \% 2 F j m c c .1996 .0251$ )

8. Cucoranu I, Clempus R, Dikalova A, Phelan PJ, Ariyan S, Dikalov S, Sorescu D (2009) $\mathrm{NAD}(\mathrm{P}) \mathrm{H}$ oxidase 4 mediates transforming growth factor-beta1induced differentiation of cardiac fibroblasts into myofibroblasts. Circ Res 97:900-907. https://doi.org/10.1161/01.RES.0000187457.24338.3D (https://doi.org/10.1161/01.RES.0000187457.24338.3D)

CrossRef (https://doi.org/10.1161/01.RES.0000187457.24338.3D)

Google Scholar (http://scholar.google.com/scholar_lookup? title=NAD\%28P\%29\%20H\%20oxidase\%204\%2omediates\%2otransforming\%2c beta1-

induced\%2odifferentiation\%20of\%20cardiac\%2ofibroblasts\%2ointo\%2omyofit 907\&publication_year=2009\&doi=10.1161\%2F01.RES.0000187457.24338.3D)

9. De Caterina R, Libby P, Peng HB, Thannickal VJ, Rajavashisth TB, Gimbrone MA Jr et al (1995) Nitric oxide decreases cytokine-induced endothelial activation. Nitric oxide selectively reduces endothelial expression of adhesion molecules and proinflammatory cytokines. J Clin Invest 96(1):60-68. https://doi.org/10.1172/JCI118074 (https://doi.org/10.1172/JCI118074) CrossRef (https://doi.org/10.1172/JCI118074) Google Scholar (http://scholar.google.com/scholar_lookup? title=Nitric\%20oxide\%20decreases\%20cytokineinduced\%20endothelial\%20activation.\%20Nitric\%200xide\%20selectively\%2ort 68\&publication_year=1995\&doi=10.1172\%2FJCI118074）

10. D'Souza SP, Davis M, Baxter GF (2004) Autocrine and paracrine actions of natriuretic peptides in the heart. Pharmacol Ther 101(2):113-129. https://doi.org/10.1016/j.pharmthera.2003.11.001 (https://doi.org/10.1016/j.pharmthera.2003.11.001) CrossRef (https://doi.org/10.1016/j.pharmthera.2003.11.001) Google Scholar (http://scholar.google.com/scholar_lookup? title=Autocrine\%20and\%2oparacrine\%20actions\%20of\%2onatriuretic\%2opept 129\&publication_year=2004\&doi=10.1016\%2Fj.pharmthera.2003.11.001)

11. Dubois G (1996) Decreased L-arginine-nitric oxide pathway in cultured myoblasts from spontaneously hypertensive versus normotensive Wistar-Kyoto rats. FEBS Lett 392(3):242-244

CrossRef (https://doi.org/10.1016/0014-5793(96)00817-4)

Google Scholar (http://scholar.google.com/scholar_lookup? title=Decreased\%20L-argininenitric\%20oxide\%2opathway\%20in\%20cultured\%20myoblasts\%20from\%20spo Kyoto\%2orats\&author=G.\%20Dubois\&journal=FEBS\%20Lett\&volume=392\&is 244\&publication_year=1996)

12. Giani JF, Miquet JG, Muñoz MC, Burghi V, Toblli JE, Masternak MM, Kopchick JJ, Bartke A, Turyn D, Dominici FP (2012) Upregulation of the angiotensin-converting enzyme 2/angiotensin-(1-7)/Mas receptor axis in the heart and the kidney of growth hormone receptor knock-out mice. Growth Hormon IGF Res 22(6):224-233. https://doi.org/10.1016/j.ghir.2012.08.003 (https://doi.org/10.1016/j.ghir.2012.08.003) CrossRef (https://doi.org/10.1016/j.ghir.2012.08.003) 
Google Scholar (http://scholar.google.com/scholar_lookup?

title=Upregulation\%20of\%20the\%20angiotensin-

converting\%20enzyme\%202\%2Fangiotensin-\%281-7\%29\%2FMas\%2Oreceptor\%20axi out\%2omice\&author=JF.\%20Giani\&author=JG.\%20Miquet\&author=MC.\%20Mu\%C3 233\&publication_year=2012\&doi $=10.1016 \% 2 F j$.ghir.2012.08.003)

13. Gonzalez W, Fontaine V, Pueyo ME, Laquay N, Messika-Zeitoun D, Philippe M, Arnal JF, Jacob MP, Michel JB (2000) Molecular plasticity of vascular wall during N(G)-nitro-L-arginine methyl ester-induced hypertension: modulation of proinflammatory signals. Hypertension 36(1):103-109

CrossRef (https://doi.org/10.1161/01.HYP.36.1.103)

Google Scholar (http://scholar.google.com/scholar_lookup?

title=Molecular\%2oplasticity\%20of\%20vascular\%20wall\%2oduring\%20N\%28C nitro-L-arginine\%20methyl\%20ester-

induced\%2ohypertension\%3A\%2omodulation\%20of\%2oproinflammatory\%20s Zeitoun\&author=M.\%20Philippe\&author=JF\%CC\%A7.\%20Arnal\&author=MP.S 109\&publication_year=2000)

14. Intengan HD, Schiffrin EL (2001) Vascular remodeling in hypertension: roles of apoptosis, inflammation, and fibrosis. Hypertension 38(3 Pt 2):581-587

CrossRef (https://doi.org/10.1161/hyogt1.096249)

Google Scholar (http://scholar.google.com/scholar_lookup?

title=Vascular\%2oremodeling\%20in\%2ohypertension\%3A\%2oroles\%20of\%20: 587\&publication_year=2001)

15. Itoh T, Nagaya N, Murakami S, Fujii T, Iwase T, Ishibashi-Ueda H, Yutani C, Yamagishi M, Kimura H, Kangawa K (2004) C-type natriuretic peptide ameliorates monocrotaline-induced pulmonary hypertension in rats. Am J Respir Crit Care Med 170(11):1204-1211. https://doi.org/10.1164/rccm.200404-455OC

(https://doi.org/10.1164/rcem.200404-455OC)

CrossRef (https://doi.org/10.1164/rccm.200404-455OC)

Google Scholar (http://scholar.google.com/scholar_lookup?

title=C-type\%2onatriuretic\%2opeptide\%20ameliorates\%20monocrotalineinduced\%2opulmonary\%2ohypertension\%20in\%2orats\&author=T.\%20Itoh\&al Ueda\&author=C.\%20Yutani\&author=M.\%20Yamagishi\&author=H.\%20Kimura 1211\&publication_year $=2004 \& d o i=10.1164 \% 2 F r c m .200404-455$ OC)

16. Jiang F, Liu GS, Dusting GJ, Chan EC (2014) NADPH oxidase-dependent redox signaling in TGF- $\beta$-mediated fibrotic responses. Redox Biol 2:267-272.

https://doi.org/10.1016/j.redox.2014.01.012

(https://doi.org/10.1016/j.redox.2014.01.012)

CrossRef (https://doi.org/10.1016/j.redox.2014.01.012)

Google Scholar (http://scholar.google.com/scholar_lookup?

title=NADPH\%20oxidase-

dependent\%2oredox\%2osignaling\%20in\%20TGF-\%CE\%B2-

mediated\%2ofibrotic\%2oresponses\&author=F.\%2OJiang\&author=GS.\%2OLiu\& 272\&publication_year $=2014 \&$ doi $=10.1016 \% 2 F j . r e d o x .2014 .01 .012)$

17. Kelsall CJ, Chester AH, Sarathchandra P, Singer DRJ (2006) Expression and localization of C-type natriuretic peptide in human vascular smooth muscle cells. Vasc Pharmacol 45:368-373. https://doi.org/10.1016/j.vph.2006.06.011 (https://doi.org/10.1016/j.vph.2006.06.011) CrossRef (https://doi.org/10.1016/j.vph.2006.06.011) 
Google Scholar (http://scholar.google.com/scholar_lookup?

title=Expression\%20and\%2olocalization\%20of\%20C-

type\%20natriuretic\%20peptide\%20in\%20human\%20vascular\%20smooth\%20muscles 373\&publication_year=2006\&doi=10.1016\%2Fj.vph.2006.06.011)

18. Leong XF, Ng CY, Jaarin K (2015) Animal models in cardiovascular research: hypertension and atherosclerosis. Biomed Res Int 2015:528757.

https://doi.org/10.1155/2015/528757 (https://doi.org/10.1155/2015/528757)

CrossRef (https://doi.org/10.1155/2015/528757)

Google Scholar (http://scholar.google.com/scholar_lookup?

title=Animal\%20models\%20in\%20cardiovascular\%2oresearch\%3A\%20hyperte

19. Li Y, Sarkar O, Brochu M, Anand-Srivastava MB (2014) Natriuretic peptide receptor-C attenuates hypertension in spontaneously hypertensive rats: role of nitroxidative stress and Gi proteins. Hypertension 63(4):846-855.

https://doi.org/10.1161/HYPERTENSIONAHA.113.01772

(https://doi.org/10.1161/HYPERTENSIONAHA.113.01772)

CrossRef (https://doi.org/10.1161/HYPERTENSIONAHA.113.01772)

Google Scholar (http://scholar.google.com/scholar_lookup?

title=Natriuretic\%2opeptide\%2oreceptor-C\%20attenuates\%2ohypertension\%2 Srivastava\&journal $=$ Hypertension $\&$ volume $=63 \&$ issue $=4 \&$ pages $=846-$

855\&publication_year $=2014 \& d o i=10.1161 \% 2 F H Y P E R T E N S I O N A H A .113 .01772$

20. Liang C, Zhang Y, Zhuo D, Lo CY, Yu L, Lau CW, Kwan YW, Tse G, Huang Y, Yao X (2019) Endothelial cell transient receptor potential channel C5 (TRPC5) is essential for endothelium-dependent contraction in mouse carotid arteries. Biochem Pharmacol 159:11-24. https://doi.org/10.1016/j.bcp.2018.11.002 (https://doi.org/10.1016/j.bcp.2018.11.002)

CrossRef (https://doi.org/10.1016/j.bcp.2018.11.002)

Google Scholar (http://scholar.google.com/scholar_lookup?

title=Endothelial\%20cell\%2otransient\%2oreceptor\%2opotential\%20channel\%s dependent\%20contraction\%2oin\%2omouse\%20carotid\%20arteries\&author=C. 24\&publication_year $=2019 \& d o i=10.1016 \% 2 F j . b c p .2018 .11 .002)$

21. Magnani ND, Marchini T, Vanasco V, Tasat DR, Alvarez S, Evelson P (2013)

Reactive oxygen species produced by NADPH oxidase and mitochondrial dysfunction in lung after an acute exposure to residual oil fly ashes. Toxicol Appl Pharmacol 270(1):31-38. https://doi.org/10.1016/j.taap.2013.04.002 (https://doi.org/10.1016/j.taap.2013.04.002)

CrossRef (https://doi.org/10.1016/j.taap.2013.04.002)

Google Scholar (http://scholar.google.com/scholar_lookup?

title=Reactive\%20oxygen\%2ospecies\%20produced\%20by\%20NADPH\%20oxid: 38\&publication_year $=2013 \& d o i=10.1016 \% 2 F j$.taap.2013.04.002)

22. Mangiafico S, Costello-Boerrigter LC, Andersen IA, Cataliotti A, Burnett JC Jr (2013) Neutral endopeptidase inhibition and the natriuretic peptide system: an evolving strategy in cardiovascular therapeutics. Eur Heart J 34(12):886-893c. https://doi.org/10.1093/eurheartj/ehs262 (https://doi.org/10.1093/eurheartj/ehs262)

CrossRef (https://doi.org/10.1093/eurheartj/ehs262)

Google Scholar (http://scholar.google.com/scholar_lookup?

title=Neutral\%20endopeptidase\%2oinhibition\%20and\%20the\%2onatriuretic\%: Boerrigter\&author=IA.\%20Andersen\&author=A.\%20Cataliotti\&author=JC.\%2C 893c\&publication_year=2013\&doi=10.1093\%2Feurheartj\%2Fehs262) 
23. Marchini T, Magnani ND, Paz ML, Vanasco V, Tasat D, González Maglio DH, Alvarez S, Evelson PA (2014) Time course of systemic oxidative stress and inflammatory response induced by an acute exposure to Residual Oil Fly Ash. Toxicol Appl Pharmacol 274(2):274-282. https://doi.org/10.1016/j.taap.2013.11.013

(https://doi.org/10.1016/j.taap.2013.11.013)

CrossRef (https://doi.org/10.1016/j.taap.2013.11.013)

Google Scholar (http://scholar.google.com/scholar_lookup?

title=Time\%20course\%20of\%20systemic\%20oxidative\%20stress\%20and\%2oin 282\&publication_year $=2014 \&$ doi $=10.1016 \% 2 F j . t a a p .2013 .11 .013)$

24. McCaffrey TA (2000) TGF-betas and TGF-beta receptors in atherosclerosis.

Cytokine Growth Factor Rev 11(1-2):103-114

CrossRef (https://doi.org/10.1016/S1359-6101(99)oo034-9)

Google Scholar (http://scholar.google.com/scholar_lookup?title=TGF-

betas\%20and\%20TGF-

beta\%20receptors\%20in\%20atherosclerosis\&author=TA.\%20McCaffrey\&journ:

114\&publication_year=2000)

25. Moyes AJ, Khambata RS, Villar I, Bubb KJ, Baliga RS, Lumsden NG, Xiao F, Gane PJ, Rebstock AS, Worthington RJ, Simone MI, Mota F, Rivilla F, Vallejo S, Peiró C, Sánchez Ferrer CF, Djordjevic S, Caulfield MJ, MacAllister RJ, Selwood DL, Ahluwalia A, Hobbs AJ (2014) Endothelial C-type natriuretic peptide maintains vascular homeostasis. J Clin Invest 124(9):4039-4051. https://doi.org/10.1172/JCI74281 (https://doi.org/10.1172/JCI74281)

CrossRef (https://doi.org/10.1172/JCI74281)

Google Scholar (http://scholar.google.com/scholar_lookup?

title=Endothelial\%20C-

type\%2onatriuretic\%2opeptide\%2omaintains\%2ovascular\%2ohomeostasis\&au 4051\&publication_year $=2014 \&$ doi $=10.1172 \% 2 F J C I 74281$ )

26. Osterbur K, Yu DH, DeClue AE (2013) Interleukin-1 $\beta$, tumor necrosis factor- $\alpha$ and lipopolysaccharide induce $\mathrm{C}$-type natriuretic peptide from canine aortic endothelial cells. Res Vet Sci 94(3):478-483.

https://doi.org/10.1016/j.rvsc.2012.10.002

(https://doi.org/10.1016/j.rvsc.2012.10.002)

CrossRef (https://doi.org/10.1016/j.rvsc.2012.10.002)

Google Scholar (http://scholar.google.com/scholar_lookup?

title=Interleukin-1\%CE\%B2\%2C\%2otumor\%2onecrosis\%2ofactor-\%CE\%B1\%2 type\%20natriuretic\%2opeptide\%20from\%20canine\%20aortic\%2oendothelial\% 483\&publication_year $=2013 \&$ doi $=10.1016 \% 2 F j . r v s c .2012 .10 .002)$

27. Piech A, Dessy C, Havaux X, Feron O, Balligand JL (2003) Differential regulation of nitric oxide synthases and their allosteric regulators in heart and vessels of hypertensive rats. Cardiovasc Res 57(2):456-467

CrossRef (https://doi.org/10.1016/Sooo8-6363(02)00676-4)

Google Scholar (http://scholar.google.com/scholar_lookup?

title=Differential\%2oregulation\%20of\%20nitric\%200xide\%20synthases\%20anc 467\&publication_year=2003)

28. Pieraggi M, Nejjar I, Julian M, Bouissou H (1986) Staining of elastic tissue by Verhoeff's iron hematoxylin. Ann Pathol 6(1):74-77

Google Scholar (http://scholar.google.com/scholar_lookup? title=Staining\%20of\%20elastic\%20tissue\%20by\%2oVerhoeff\%E2\%80\%99s\%2C 77\&publication_year=1986) 
29. Pollman MJ, Naumovski L, Gibbons GH (1999) Vascular cell apoptosis: cell type-specific modulation by transforming growth factor-beta1 in endothelial cells versus smooth muscle cells. Circulation 99:2019-2026

CrossRef (https://doi.org/10.1161/01.CIR.99.15.2019) Google Scholar (http://scholar.google.com/scholar_lookup? title=Vascular\%20cell\%20apoptosis\%3A\%20cell\%2otypespecific\%20modulation\%2oby\%20transforming\%2ogrowth\%2ofactorbeta1\%20in\%2oendothelial\%20cells\%2oversus\%20smooth\%2omuscle\%20cells 2026\&publication_year=1999)

30. Qian JY, Haruno A, Asada Y, Nishida T, Saito Y, Matsuda T, Ueno H (2002) Local expression of C-type natriuretic peptide suppresses inflammation, eliminates shear stress-induced thrombosis, and prevents neointima formation through enhanced nitric oxide production in rabbit injured carotid arteries. Circ Res 91(11):1063-1069

CrossRef (https://doi.org/10.1161/01.RES.0000043631.25915.E6)

Google Scholar (http://scholar.google.com/scholar_lookup? title=Local\%20expression\%20of\%20Ctype\%20natriuretic\%2opeptide\%20suppresses\%2oinflammation\%2C\%2oelimiı induced\%2othrombosis\%2C\%20and\%2oprevents\%2oneointima\%2oformation? 1069\&publication_year=2002)

31. Romero M, Caniffi C, Bouchet G, Elesgaray R, McLaughlin M, Tomat A et al (2013) Sex differences in the beneficial cardiac effects of chronic treatment with atrial natriuretic peptide in spontaneously hypertensive rats. PLoS One 8

(8):e71992. https://doi.org/10.1371/journal.pone.0071992

(https://doi.org/10.1371/journal.pone.0071992)

CrossRef (https://doi.org/10.1371/journal.pone.0071992)

Google Scholar (http://scholar.google.com/scholar_lookup?

title=Sex\%20differences\%20in\%20the\%2obeneficial\%20cardiac\%2oeffects\%20

32. Ruiz-Ortega M, Rodríguez-Vita J, Sanchez-Lopez E, Carvajal G, Egido J (2007)

TGF-beta signaling in vascular fibrosis. Cardiovasc Res 74(2):196-206.

https://doi.org/10.1016/j.cardiores.2007.02.008

(https://doi.org/10.1016/j.cardiores.2007.02.008)

CrossRef (https://doi.org/10.1016/j.cardiores.2007.02.008)

Google Scholar (http://scholar.google.com/scholar_lookup?title=TGFbeta\%20signaling\%20in\%20vascular\%2ofibrosis\&author=M.\%2oRuizOrtega\&author=J.\%20Rodr\%C3\%ADguez-Vita\&author=E.\%20SanchezLopez\&author=G.\%20Carvajal\&author=J.\%20Egido\&journal=Cardiovasc\%20R 206\&publication_year $=2007 \&$ doi $=10.1016 \% 2 F j$.cardiores.2007.02.008)

33. Saha S, Li Y, Lappas G, Anand-Srivastava MB (2008) Activation of natriuretic peptide receptor-C attenuates the enhanced oxidative stress in vascular smooth muscle cells from spontaneously hypertensive rats: implication of $\mathrm{Gi}$ alpha protein. J Mol Cell Cardiol 44:336-344. https://doi.org/10.1016/j.yjmcc.2007.11.003

(https://doi.org/10.1016/j.yjmcc.2007.11.003)

CrossRef (https://doi.org/10.1016/j.yjmcc.2007.11.003)

Google Scholar (http://scholar.google.com/scholar_lookup? title=Activation\%20of\%2onatriuretic\%2opeptide\%2oreceptor-C\%20attenuates' Srivastava\&journal=J\%20Mol\%20Cell\%20Cardiol\&volume $=44 \&$ pages $=336-$ 344\&publication_year=2008\&doi=10.1016\%2Fj.yjmcc.2007.11.003) 
Sanz-Rosa D, Oubiña MP, Cediel E, de las Heras N, Vegazo O, Jiménez J, Lahera V, Cachofeiro V (2005) Effect of AT1 receptor antagonism on vascular and circulating inflammatory mediators in SHR: role of NF- $\mathrm{kB} / \mathrm{I} \kappa \mathrm{B}$ system. Am J Physiol Heart Circ Physiol 288:H111-H115. https://doi.org/10.1152/ajpheart.01061.2003

(https://doi.org/10.1152/ajpheart.01061.2003)

CrossRef (https://doi.org/10.1152/ajpheart.01061.2003)

Google Scholar (http://scholar.google.com/scholar_lookup?

title=Effect\%20of\%20AT1\%2oreceptor\%20antagonism\%20on\%20vascular\%20and\%2 Rosa\&author=MP.\%20Oubi\%C3\%B1a\&author=E.\%2oCediel\&author=N.\%2olas\%20H H115\&publication_year=2005\&doi=10.1152\%2Fajpheart.01061.2003)

35. Schiffrin EL (2012) Vascular remodeling in hypertension: Mechanisms and Treatment. Hypertension 59:367-374. https://doi.org/10.1161/HYPERTENSIONAHA.111.187021 (https://doi.org/10.1161/HYPERTENSIONAHA.111.187021)

36. Sprague AH, Khalil RA (2009) Inflammatory cytokines in vascular dysfunction and vascular disease. Biochem Pharmacol 78:539-552.

https://doi.org/10.1016/j.bcp.2009.04.029

(https://doi.org/10.1016/j.bcp.2009.04.029)

CrossRef (https://doi.org/10.1016/j.bcp.2009.04.029)

Google Scholar (http://scholar.google.com/scholar_lookup?

title=Inflammatory\%20cytokines\%20in\%20vascular\%2odysfunction\%20and\%: 552\&publication_year $=2009 \& d o i=10.1016 \% 2 F j . b c p .2009 .04 .029)$

37. Stingo AJ, Clavell AL, Heublein DM, Wei CM, Pittelkow MR, Burnett JCJ (1992) Presence of C-type natriuretic peptide in cultured human endothelial cells and plasma. Am J Phys 263:H1318-H1321.

https://doi.org/10.1152/ajpheart.1992.263.4.H1318

(https://doi.org/10.1152/ajpheart.1992.263.4.H1318)

Google Scholar (http://scholar.google.com/scholar_lookup?

title $=$ Presence $\% 200 f \% 20 C-$

type\%2onatriuretic\%2opeptide\%2oin\%2ocultured\%2ohuman\%2oendothelial\%

H1321\&publication_year=1992\&doi=10.1152\%2Fajpheart.1992.263.4.H1318)

38. Vapaatalo H, Mervaala E, Nurminen ML (2000) Role of endothelium and nitric oxide in experimental hypertension. Physiol Res 49(1):1-10

Google Scholar (https://scholar.google.com/scholar?

q=Vapaatalo\%20H\%2C\%2oMervaala\%20E\%2C\%20Nurminen\%20ML\%20\%28

39. Vardeny O, Miller R, Solomon SD (2014) Combined neprilysin and reninangiotensin system inhibition for the treatment of heart failure. J Am Coll Cardiol HF (14)00338-2. https://doi.org/10.1016/j.jchf.2014.09.001 (https://doi.org/10.1016/j.jchf.2014.09.001)

40. Vaziri ND, Ni Z, Oveisi F (1998) Upregulation of renal and vascular nitric oxide synthase in young spontaneously hypertensive rats. Hypertension 31(6):1248 $-1254$ CrossRef (https://doi.org/10.1161/01.HYP.31.6.1248)

Google Scholar (http://scholar.google.com/scholar_lookup? title=Upregulation\%20of\%20renal\%20and\%20vascular\%20nitric\%20oxide\%2C 1254\&publication_year=1998)

41. Wagenseil JE, Mecham RP (2009) Vascular extracellular matrix and arterial mechanics. Physiol Rev 89(3):957-989. 
https://doi.org/10.1152/physrev.00041.2008

(https://doi.org/10.1152/physrev.00041.2008)

CrossRef (https://doi.org/10.1152/physrev.00041.2008)

Google Scholar (http://scholar.google.com/scholar_lookup?

title=Vascular\%20extracellular\%20matrix\%20and\%20arterial\%2omechanics\&author=

989\&publication_year=2009\&doi=10.1152\%2Fphysrev.00041.2008)

\section{Copyright information}

(C) Springer-Verlag GmbH Germany, part of Springer Nature 2019

\section{About this article}

Cite this article as:

Caniffi, C., Cerniello, F.M., Bouchet, G. et al. Pflugers Arch - Eur J Physiol (2019). https://doi.org/10.1007/s00424-019-02287-y

- Received 30 January 2019

- Revised 20 May 2019

- Accepted 22 May 2019

- First Online 11 June 2019

- DOI https://doi.org/10.1007/s00424-019-02287-y

- Publisher Name Springer Berlin Heidelberg

- Print ISSN 0031-6768

- Online ISSN 1432-2013

- About this journal

- Reprints and Permissions

\section{Personalised recommendations}

(C) 2018 Springer Nature Switzerland AG. Part of Springer Nature.

Not logged in Not affiliated 181.47.11.55 